\title{
Reactive Power Support Adequacy at the DSO/TSO Interface
}

\author{
Stefan Stanković \\ Lennart Söder \\ Division of Electric Power and Energy Systems \\ KTH Royal Institute of Technology \\ Stockholm, Sweden \\ \{stanko, 1sod\}@kth.se
}

\author{
Zita Hagemann \\ Christian Rehtanz \\ ie $^{3}$ Institute \\ TU Dortmund University \\ Dortmund, Germany \\ \{zita.hagemann, christian.rehtanz\}@tu-dortmund.de
}

\begin{abstract}
The exchange of reactive power at the interface between distribution and transmission systems gains significant importance with ongoing relocation of generators to lower voltage levels. To keep transmission systems operation secure and stable, distribution systems may need to be more flexible when exchanging power at the interface with the overlaying grid. A flexibility measure of this interface is proposed by this paper through introduction of flexibility coefficients. These coefficients are calculated by comparing DSO capability and TSO desirability surfaces. These surfaces describe the distribution system's capability to provide flexible reactive power exchange and the transmission system's needs to use these flexibilities. Since the surfaces can be calculated independently, the newly proposed method of flexibility assessment can be applied without substantial exchange of system information between DSO and TSO. The proposed concept is illustrated on the Nordic 32 system for a case of the connection of two differently behaving distribution systems.
\end{abstract}

Index Terms-Active Distribution Networks, Reactive Power Support, Flexibility Coefficients, Transmission Systems, Voltage Stability and Security

\section{INTRODUCTION}

The continuing change in the energy mix towards more renewables leads to relocation of active and reactive power generating sources from transmission grids to lower voltage levels. As a consequence, transmission systems may not have any longer access to the synchronous generators, the main suppliers of reactive power. Without synchronous generators to provide stable supply of reactive power, transmission systems will face challenges keeping the voltages in desired bounds and assuring voltage stability of the system. One of the solutions would be to supply necessary reactive power from connected active distribution networks (ADN) [1]. In this case, transmission system operators (TSO) will request reliable information on how much flexibilities in reactive power injections the distribution system operator (DSO) can offer.

The importance of accurate interaction of the DSOs and TSOs on stable operation of the system has been pointed out by a number of institutions [2]-[6]. The ENTSO-E reviewed this profound change in [3]-[5] by describing the expected challenges and guiding the grid operators in their interaction pointing out the importance of the flexible operations on DSO/TSO interfaces. The European commission issued a demand connection code [6] guiding European TSOs to procure active and reactive power services from connected DSOs by allowing greater flexibilities of active and reactive power exchange at the point of common coupling (PCC). Flexibilities and procurement of ancilary services at the PCC from market perspective have been analyzed in [7], [8].

Numerical [9]-[16] and analytical [17] approaches have been used to assess active and reactive power exchange flexibilities for a number of case studies taking into account different criteria and operating conditions. Common to all of them is that the flexibilities have been analyzed from the DSO point of view. These flexibilities have been commonly defined as a range of values for active and reactive power at the PCC obtained from the economically and technically feasible operating conditions of the ADN [9]-[13]. The described flexibilities are very often referred as flexibilities at DSO/TSO interface.

However, the flexibility of DSO/TSO interface does not only depend on ADN's reactive power exchange behavior but also on transmission system ability to accept that exchange. Therefore, this paper proposes a new method to more precisely describe this flexibility taking into account a DSO's as well as TSO's point of view.

By finding a trade off between ADN's capability to adjust its power exchange behavior and transmission system needs for such adjustments, we introduce two flexibility coefficients: $D_{\text {flex }}$ and $T_{\text {flex }}$. The first coefficient is telling to what extent the ADN's flexibility can be used for transmission system needs. The second describes how valuable these flexibilities are for the TSO. Both coefficients are calculated using introduced DSO capability surface and TSO desirability surface. The first surface corresponds to the flexibility areas previously described in the literature [9]-[13]. The second surface, as proposed by this paper, can be obtained through the optimization routine resulting in a set of active and reactive power exchange points that satisfy TSO criteria for safe and economical operation of the transmission system. TSO desirability surface can also be given through more conservative but simpler defined bounds as described in most grid codes with demand connection rules [6], [18], [19]. 
Both mentioned surfaces can be obtained independently from the knowledge of the other system's model or behavior. Therefore, in DSO/TSO interaction, the information that would need to be exchanged is only the mentioned surfaces. This means that our method does not need exchange of sensitive system information data to provide substantial knowledge about flexibility of the DSO/TSO interface. As such, it may be used in Local Ancillary Service Market or Shared Balancing Responsibility model for DSO/TSO coordination proposed by [20]. The proposed method provides a good way for both systems to acquire the useful information for their operation without jeopardizing each others privacy.

The importance of DSO/TSO cooperation and use of reactive power flexibilities at their interface will increase with higher shares of renewables in the system and more stochastic power flows in the grid [1]. To use these flexibilities, both DSOs and TSOs should know the availability of those and their impact. This paper provides a new way to supply that information.

\section{FleXibility COEFFICIENTS}

While depending on the loading situation in an ADN, reactive and active power exchange at the DSO/TSO interface affects secure operation of the overlaying transmission system. Therefore, TSOs commonly require from DSOs to adequately adapt their power exchange behavior such that it matches a desired operation of the transmission grid. These requirements are usually reflected in the grid codes and also actively discussed in the industry and scientific community [6], [7], [21], [22].

In order to meet these standards, ADNs might need to provide enhanced power flexibility at the interface with the overlaying grid in the future. A measure on how flexible ADNs are at the specific connection point is given in this paper through introduction of flexibility coefficients. In order to define these flexibility coefficients, DSO capability surface and TSO desirability surface need to be defined first.

\section{A. DSO capability surface}

A range in which an ADN can exchange active and reactive power with an overlaying grid has been widely studied in the literature [9]-[13]. This range is usually a result of non-controllable active power behavior of renewables and loads and controllable reactive power actions of distributed generators and possibly some loads hosted in the ADN. All the definitions of this range can be encompassed with the definition of, what we call in this paper, DSO capability surface:

Definition 1. A DSO capability surface $\mathcal{D}$ is a set of points in the $(P, Q)$ plane representing active $\left(P_{p c c}\right)$ and reactive power $\left(Q_{p c c}\right)$ values at the DSO/TSO interface for each operating state of the distribution system satisfying distribution system constraints.
The aforementioned definition describes a surface of $\left(P_{p c c}\right.$, $Q_{p c c}$ ) values without any assumptions on controllability of $P_{p c c}$ and $Q_{p c c}$. In this paper, we will assume controllability of $Q_{p c c}$ while $P_{p c c}$ is assumed to have a stochastic, noncontrollable behavior coming from the nature of distributed renewable generation and loads hosted in the ADN. Given this assumption, lower $Q_{p c c}^{\min }\left(P_{p c c}\right)=\min \left(Q_{p c c}\right)$ and upper $Q_{p c c}^{\max }\left(P_{p c c}\right)=\max \left(Q_{p c c}\right)$ bound of the DSO capability surface can be found by solving a sequence of problems (1) for different values of active power generation and consumption in the grid:

$$
\begin{array}{cl}
\min / \max & Q_{p c c} \\
\text { s.t. } & \sum_{l} P_{i l}^{i n j}=g_{P i}^{d}\left(x_{d}, p_{d}\right), \forall i \in \text { DBus, } \\
& \sum_{l} Q_{i l}^{i n j}=g_{Q i}^{d}\left(x_{d}, p_{d}\right), \forall i \in \text { DBus, } \\
& \underline{U}_{i}<U_{i}<\bar{U}_{i}, \forall i \in \mathrm{DBus}, \\
& \underline{Q}_{j}<Q_{j}<\bar{Q}_{j}, \forall j \in \mathrm{DG},
\end{array}
$$

where DBus is a set of ADN's buses and DG $\subset$ DBus is a set of distributed generators hosted in the ADN. The equality constraints comprise the power balance at each bus of the ADN. The injected active and reactive powers with unique index $l$ at bus $i$ are denoted with $P_{i l}^{i n j}$ and $Q_{i l}^{i n j}$ while $g_{P i}^{d}\left(x_{d}, p_{d}\right)$ and $g_{Q i}^{d}\left(x_{d}, p_{d}\right)$ are expressions defining powers injected from the bus $i$ into the lines. Since the active powers of the loads and generators are non-controllable, they are given as parameters in (1). The decision variables of (1) are reactive powers of the generators $Q_{j}$. The reactive power exchange at the PCC, $Q_{p c c}=f\left(x_{d}, p_{d}\right)$ is a multi-variable, nonlinear function of ADN state variables $x_{d}$ and parameters $p_{d}$. The inequality constraints define physical limits of the system:

- voltage limits on the buses $\left(\underline{U}_{i}, \bar{U}_{i}\right)$

- reactive power limits of the generating units $\left(\underline{Q}_{j}, \bar{Q}_{j}\right)$

Reactive power limits $\underline{Q}_{j}, \bar{Q}_{j}$ depend on the active power of generator $P_{j}$ being considered as a constant in this optimization problem. Therefore, $\underline{Q}_{j}, \bar{Q}_{j}$ can be pre-calculated based on the value of $P_{j}$ and the generator capability charts. Depending on the type of the generator, its capability chart is given by [23]-[25].

In order to obtain the bounds $Q_{p c c}^{\min }\left(P_{p c c}\right)$ and $Q_{p c c}^{\max }\left(P_{p c c}\right)$ for the whole range of possible values for $P_{p c c}$, a realistic grid model and a dataset of all possible values for active power production and consumption in the ADN would be needed. The dataset can be obtained from an already existing timeseries describing variations in production and consumption levels or it can be created using Monte-Carlo method. DSOs may get the needed data from SCADA records for operation of the system over observed period of time.

Computational costs of solving a sequence of problems (1) depend on the choice of programming method. The results of (1) in this paper are obtained using Interior-point method [26] with problem description in MATLAB. To speed up 
the execution of the sequence, each next iteration of the optimisation problem (1) is initialized with the solution of the previous iterate. In this way, an intial point of the search is located in some vicinity of the solution since the conditions of the system are not changing substantially inbetween the iterates.

Solving (1) for the different values of production and consumption, a scatter plot data is obtained. The final bounds of DSO desirability surface are determined such that $5 \%$ of the points are allowed to be inside the surface, similarly as in [13]. Finding the scatter plot data and then fitting the bounds through them may end up to be quite computationaly heavy. Recently, some less computational demanding procedures of finding DSO capability surface have been proposed [9]. On the other hand, since DSO capability surface is supposed to be calculated offline, the procedure used in this paper is computationally acceptable.

\section{B. TSO desirability surface}

In order to be able to connect to the transmission system, one should satisfy certain requirements [6]. These requirements are usually a result of detailed studies of the impact of a certain connection on a secure operation of the transmission system. One of the outcomes of these studies is a set of values for active $P_{p c c}$ and reactive power $Q_{p c c}$ exchange at the PCC that the transmission system can accommodate without jeopardizing its secure operation. Such a set is referred in this paper as a TSO desirability surface defined below:

Definition 2. A TSO desirability surface $\mathcal{T}$ is a set of points in the $(P, Q)$ plane representing values of $\left(P_{p c c}, Q_{p c c}\right)$ pairs for which the transmission system can be operated in a secure way satisfying transmission system constraints for one or more operating points of the transmission system.

A set of allowed $\left(P_{p c c}, Q_{p c c}\right)$ pair values at the bus of interest should satisfy TSOs techno-economic requirements such as:

1) voltage stability limits,

2) bus voltage limits,

3) active power losses limits,

4) etc.

One method of finding a TSO desirability surface bounds, proposed by this paper, is to solve an optimization problem that finds utmost $\left(P_{p c c}, Q_{p c c}\right)$ pairs satisfying afore listed and possibly some other TSO criteria. Such an optimization problem can be divided into two steps. The first step would be to find utmost values $\min \left(P_{p c c}\right)$ and $\max \left(P_{p c c}\right)$ for any value of $Q_{p c c}$ subject to the constraints coming from the TSO criteria. These values will correspond to the utmost points of the surface projected on the P-axis. The second step is to find utmost values $\min \left(Q_{p c c}\right)$ and $\max \left(Q_{p c c}\right)$ for each value of
$P_{p c c} \in\left[\min \left(P_{p c c}\right), \max \left(P_{p c c}\right)\right]$. In this paper, the first step of finding a TSO desirability surface is done by solving:

$$
\begin{aligned}
& \min / \max d P \\
& \text { s.t. } \quad \sum_{l} P_{i l}^{i n j}-L_{i}^{P} \cdot d P=g_{P i}^{t}\left(x_{t}, p_{t}\right), \forall i \in \text { TBus, } \\
& \sum_{l} Q_{i l}^{i n j}-L_{i}^{Q} \cdot d Q=g_{Q i}^{t}\left(x_{t}, p_{t}\right), \forall i \in \text { TBus, } \\
& \underline{U}_{i}<U_{i}<\bar{U}_{i} \\
& \underline{q}_{j}\left(P_{j}, U_{j}\right)<Q_{j}<\bar{q}_{j}\left(P_{j}, U_{j}\right), \quad \forall j \in \mathrm{TG}, \\
& \underline{P}_{j}<P_{j}<\overline{P_{j}} \\
& \forall j \in \mathrm{TG}, \\
& P_{\text {loss }}<5 \% \text {, }
\end{aligned}
$$$$
\forall i \in \text { TBus, }(2 \mathrm{~d})
$$

where TBus is a set of transmission system buses and TG $\subset$ TBus is a set of generators connected to the transmission grid. Equality constraints (2b) and (2c) account for the balance of powers in the system and also for the voltage stability requirements of the TSO [27]. Injected active and reactive powers with unique index $l$ at bus $i$ are denoted with $P_{i l}^{i n j}$ and $Q_{i l}^{i n j}$ while $g_{P i}^{t}\left(x_{t}, p_{t}\right)$ and $g_{Q i}^{t}\left(x_{t}, p_{t}\right)$ are expressions defining powers injected from the bus $i$ into the transmission lines. Parameter vectors $L^{P}$ and $L^{Q}$ are defining the direction in which the loading of the grid is analyzed. If the increase of the load at the PCC bus $k$ is not correlated with the increase of the loads connected at other buses, $L^{P}$ and $L^{Q}$ are filled with zeros except for the position $k$ where they will have ones. If a correlation exists, then beside the nonzero element on the PCC bus position $k$, the nonzero elements would appear also in the positions corresponding to the correlated buses. Variables $d P$ and $d Q$ represent active and reactive loading levels of the transmission system.

Inequality $(2 \mathrm{~d})$ represents bus voltage limits while inequalities (2e) and (2f) represent limits coming from capability charts of the generators connected to the transmission system. It is worth mentioning that reactive power limits from (2e) depend on the current value of the generator active power $P_{j}$ as well as the value of the bus voltage $U_{j}$ to which the generator is connected. The last inequality $(2 \mathrm{~g})$ reflects the TSO's economic criterion by limiting the active power losses $P_{\text {loss }}$ in the network to $5 \%$ of the total transmitted power.

As the first step in finding the TSO desirability surface is to find utmost values of active power exchange at the PCC, objective of (2) is to minimize or maximize active power loading $d P$ at the bus of interest chosen by appropriate setting of $L^{P}$ and $L^{Q}$. Decision variables are active power loading $d P$, reactive power loading $d Q$, reactive powers of the generators $Q_{j}$, and active powers of the generators providing balancing services to the grid $P_{j}$. Limits on how much each generator can ramp up or ramp down its production to balance the powers in the system are given by (2f). States $x_{t}$ consisting of bus voltage values and bus angles are calculated to satisfy equality constraints $(2 \mathrm{~b})$ and $(2 \mathrm{c})$.

By solving $(2), \min \left(P_{p c c}\right)$ and $\max \left(P_{p c c}\right)$ are found. The second step in finding the TSO desirability surface is to find $\min \left(Q_{p c c}\right)$ and $\max \left(Q_{p c c}\right)$ for each value of $P_{p c c} \in$ 


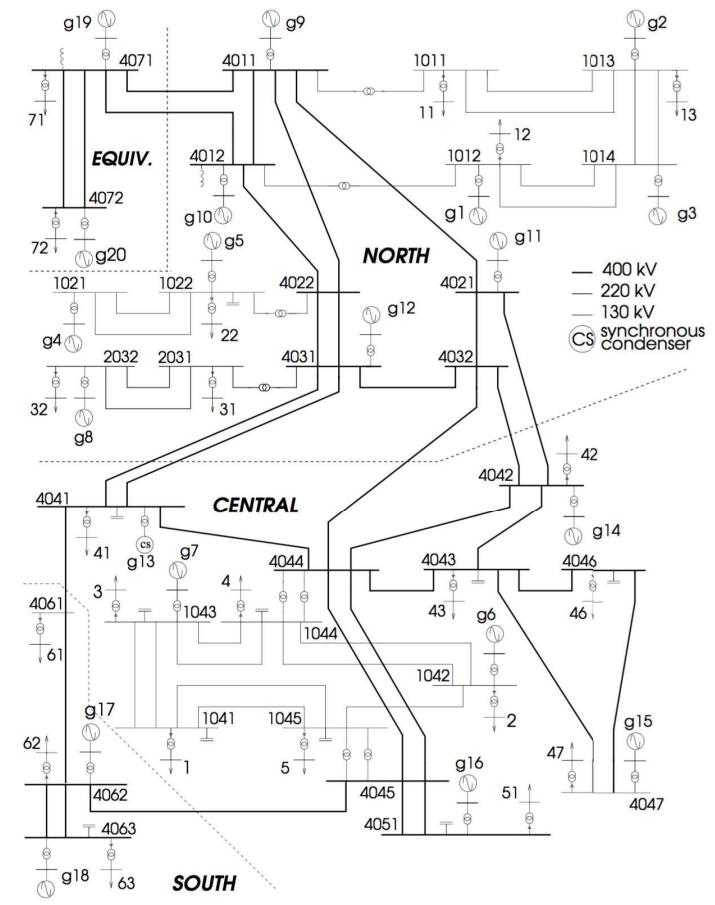

Fig. 1. Nordic 32 test system [28]

$\left[\min \left(P_{p c c}\right), \max \left(P_{p c c}\right)\right]$. This can be done with a slight modification of the problem (2). The variable $d P$ is converted to the parameter taking values from $\left[\min \left(P_{p c c}\right), \max \left(P_{p c c}\right)\right]$. The new objective is set as:

$$
\min / \max d Q
$$

with $d Q, Q_{j}$ and $P_{j}$ as decision variables. Solving the sequence of problems (3) for different values of $d P$, the lower and upper bound of TSO desirability surface are obtained.

The previously described procedure of obtaining TSO desirability surface is applied for one specific operating point of the transmission system reflected in the values of the parameters $\underline{P}_{j}, \bar{P}_{j}, P_{i l}^{i n j}$, and $Q_{i l}^{i n j}$. If the transfiguration of the network is changing, including switching of reactor/capacitor banks, the functions $g_{P i}^{t}$ and $g_{Q i}^{t}$ will change with the operating state of the system as well. Then, the TSO desirability surface satisfying multiple operating points can be found as the intersection of the TSO desirability surfaces calculated for each individual operating point of the transmission system.

\section{Calculation of Flexibility Coefficients}

A flexibility of a DSO/TSO interface can be differently evaluated depending on the perspective. A DSO may think of it as the transmission system's ability to host varying active and reactive power injection at the PCC. On the other hand, a TSO can think of the flexibility of the same bus as a capability of ADN to adjust its active and reactive power behavior to match the transmission system needs. To account for both perspectives, we introduce two flexibility coefficients $D_{\text {flex }}$ and $T_{\text {flex }}$ as a measure on how flexible a DSO/TSO interface is.

The first coefficient, quantifying flexibility of the interface from the DSO point of view, can be calculated as:

$$
D_{\text {flex }}=A(\mathcal{D} \cap \mathcal{T}) / A(\mathcal{D})
$$

where $A(\cdot)$ is the operator giving the area of the surface, $\mathcal{D}$ is DSO capability surface and $\mathcal{T}$ is TSO desirability surface.

The second coefficient, quantifying flexibility of the interface from the TSO point of view can be calculated as:

$$
T_{\text {flex }}=A(\mathcal{D} \cap \mathcal{T}) / A(\mathcal{T})
$$

\section{CASE STUdY}

In order to illustrate the calculation of the proposed flexibility coefficients, a transmission system and two candidate ADNs are chosen. As the transmission system, the Nordic 32 test system [28] shown on Fig. 1 is used. Lower $\left(\underline{U}_{i}\right)$ and upper $\left(\bar{U}_{i}\right)$ bus voltage limits are assumed to be 0.94 p.u. and 1.06 p.u. respectively. The synchronous generators connected to the system have capability charts described by [23]. It is assumed that all generators participate in the balancing operations of the system. Ramping down limit $\underline{P}_{j}$ of the generators is chosen to be $5 \%$ of the actual active power while ramping up limit $\bar{P}_{j}$ is bounded by the capability curve of the generator $j$. Generator 20 is assumed to be infinite bus representing northern interconnections of the Nordic 32 system with other systems. In order to get TSO desirability surface for a bus in the Nordic 32, (2) is solved for two loading cases of the system: $80 \%$ and $100 \%$. In the $80 \%$ loading case, both the powers of the loads and generators actual active powers are decreased by $20 \%$. The resulting TSO desirability surface is found as the intersection between these two surfaces.

Two ADNs capable of providing the flexibility are considered for connection to the transmission system:

1) modified IEEE $14+2$ test system with integrated type 4 wind turbines,

TABLE I

VAlues of $D_{\text {flex }}$ AND $T_{\text {flex }}$ AT DIFFERENT BUSES OF THE NORDIC 32 SYSTEM FOR DIFFERENT CONNECTION OF EVALUATED ADNS

\begin{tabular}{|c|c|c|c|c|c|}
\hline \multicolumn{2}{|c|}{ Nordic 32 buses } & \multicolumn{2}{|c|}{ ADN 1 } & \multicolumn{2}{c|}{ ADN 2 } \\
\hline area & name & $D_{\text {flex }}$ & $T_{\text {flex }}$ & $D_{\text {flex }}$ & $T_{\text {flex }}$ \\
\hline \multirow{4}{*}{} & 1011 & 1 & 0.0022 & 1 & 0.1158 \\
\cline { 2 - 6 } & 2032 & 1 & 0.0089 & 0.6395 & 0.3037 \\
\cline { 2 - 6 } & 4012 & 1 & 0.0009 & 1 & 0.0497 \\
\cline { 2 - 6 } & 4022 & 1 & 0.0042 & 0.8939 & 0.201 \\
\cline { 2 - 6 } & 4032 & 1 & 0.0029 & 0.9566 & 0.1472 \\
\hline \multirow{5}{*}{ 芯 } & 1041 & $/$ & $/$ & 0.2897 & 0.5446 \\
\cline { 2 - 6 } & 1043 & 1 & 0.0288 & 0.3495 & 0.5348 \\
\cline { 2 - 6 } & 1044 & 1 & 0.0076 & 0.8264 & 0.334 \\
\cline { 2 - 6 } & 1045 & 1 & 0.0119 & 0.713 & 0.4493 \\
\cline { 2 - 6 } & 4041 & 1 & 0.005 & 0.8851 & 0.2332 \\
\cline { 2 - 6 } & 4043 & 1 & 0.0052 & 0.9196 & 0.252 \\
\cline { 2 - 6 } & 4047 & 1 & 0.0071 & 0.836 & 0.3154 \\
\cline { 2 - 6 } & 4051 & 1 & 0.0139 & 0.6754 & 0.4998 \\
\hline \multirow{\Xi}{*}{} & 4061 & 1 & 0.0062 & 0.6793 & 0.2225 \\
\cline { 2 - 6 } & 4062 & 1 & 0.004 & 0.9785 & 0.2064 \\
\hline
\end{tabular}




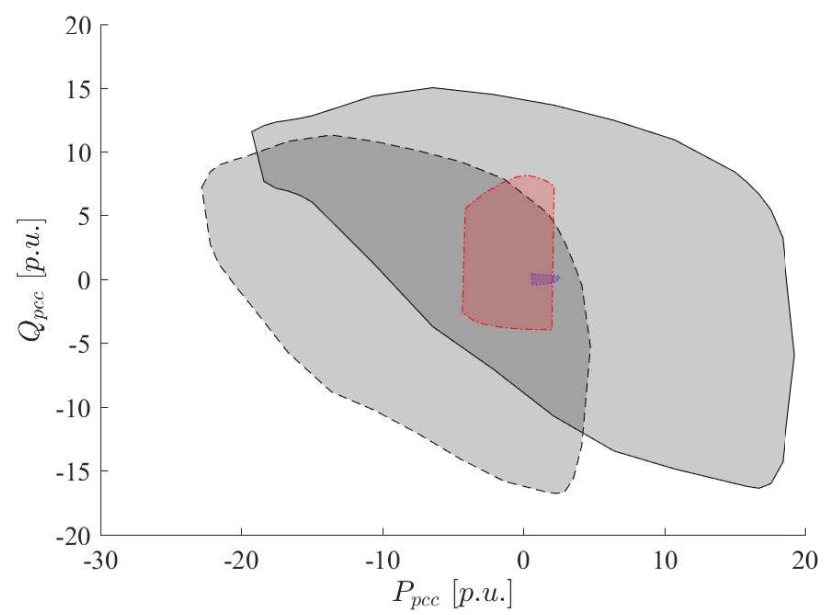

(a) Bus 4043

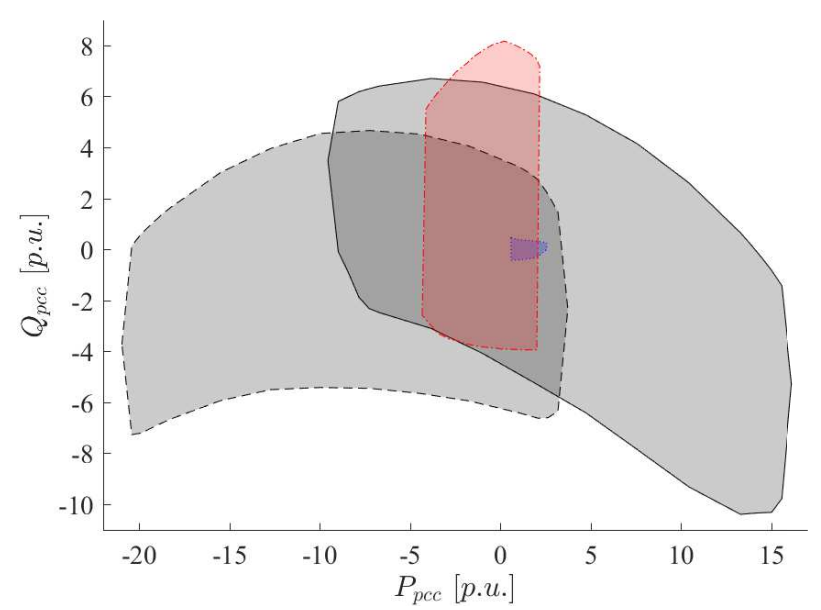

(b) Bus 4051

Fig. 2. TSO desirability surfaces for the buses in the central area with DSO capability surfaces of considered ADNs

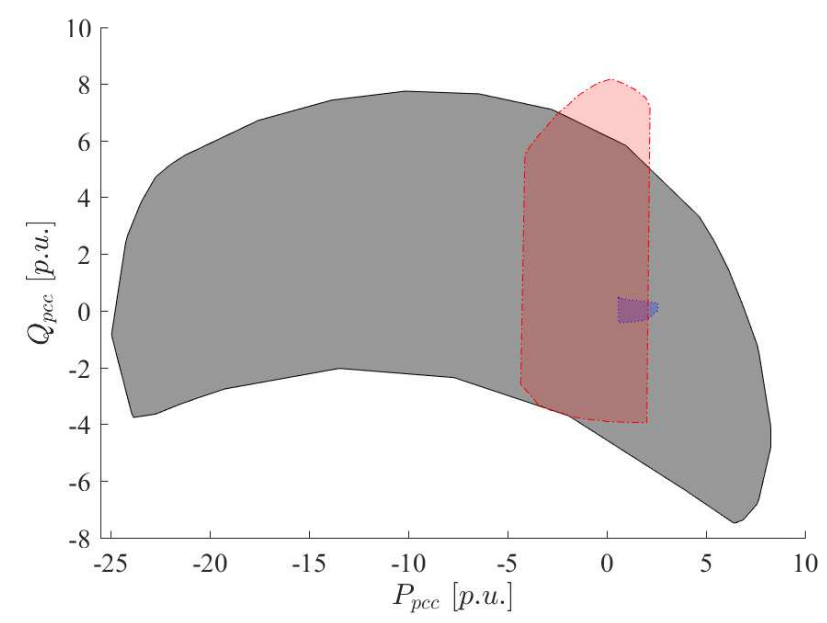

(a) Bus 4022

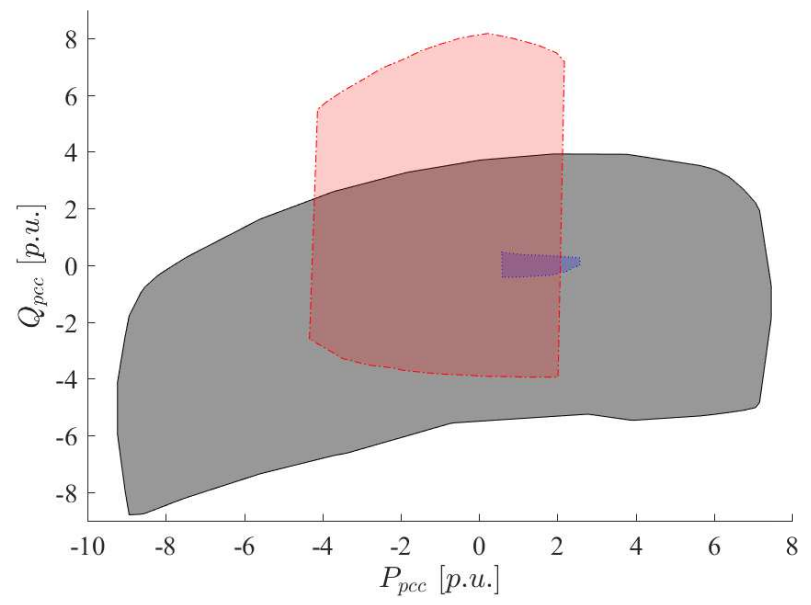

(b) Bus 2032

Fig. 3. TSO desirability surfaces for the buses in the northern area with DSO capability surfaces of considered ADNs

2) distribution system consisting of 50 IEEE 69 radial grids with integrated type 4 wind turbines.

The first ADN is a modified IEEE 14 test system with integrated type 4 wind turbines. The corresponding capability chart of these generators is implemented as in [25]. A loop is added between buses 9 and 13 containing two additional buses: bus 15 , on which a $10 \mathrm{MW}$ wind farm is connected and bus 16 on which the load $(\mathrm{P}=10 \mathrm{MW}, \mathrm{Q}=3 \mathrm{MVAr})$ is connected. The lines creating the loop have $\mathrm{R} / \mathrm{X}$ ratios equal to one. The lines connecting buses 13-15 and 16-9 have a reactance of 0.1 p.u. while the line $15-16$ has a reactance of 0.2 p.u.. Other wind farms are connected to buses 3 (50 MW), 6 (15 MW), and $10(10 \mathrm{MW})$. The generator at the bus 2 is removed. Loads at buses 3 and 4 are scaled down 50\%. Bus 1 is considered to be the PCC being connected to the transmission grid through a regulating transformer. It is assumed that the on-load tap changer (OLTC) of the transformer is capable of keeping 1 p.u. voltage on the secondary side to which the bus 1 is connected.

The second considered ADN consists of 50 IEEE 69 sys- tems connected to the same PCC bus on the secondary side of the regulating transformer. The OLTC of the transformer is assumed to be capable of keeping 1 p.u. voltage on the secondary side. Each IEEE 69 network is complemented with eight type 4 wind turbines with nominal powers ranging from 1 to $2.5 \mathrm{MW}$ connected to the buses $5,15,23,30,38,42,53$ and 60. Capability chart of each type 4 turbine is implemented as in [25]. Voltage limits in the grid are assumed to be $\pm 10 \%$. Big industrial loads whose power exceeds $0.3 \mathrm{MW}$ are assumed to have constant consumption. All reactive power loads are scaled to $30 \%$ of their original value.

In order to get different operating points of the ADNs corresponding to different values of $P_{p c c}$, variation of generation and consumption levels in ADNs is applied. The variation trend of generation and consumption in central Sweden (SE3 area) is taken from the Nordpool database [29] and interpolated with the ADNs system data in the following way:

1) Generation levels are linearly scaled to correspond to variations in Nordpool data; 


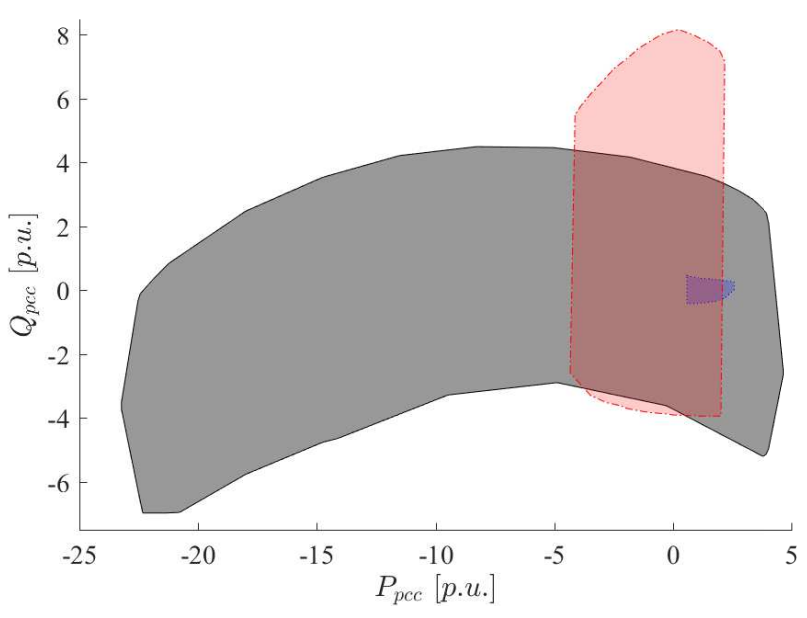

(a) Bus 4061

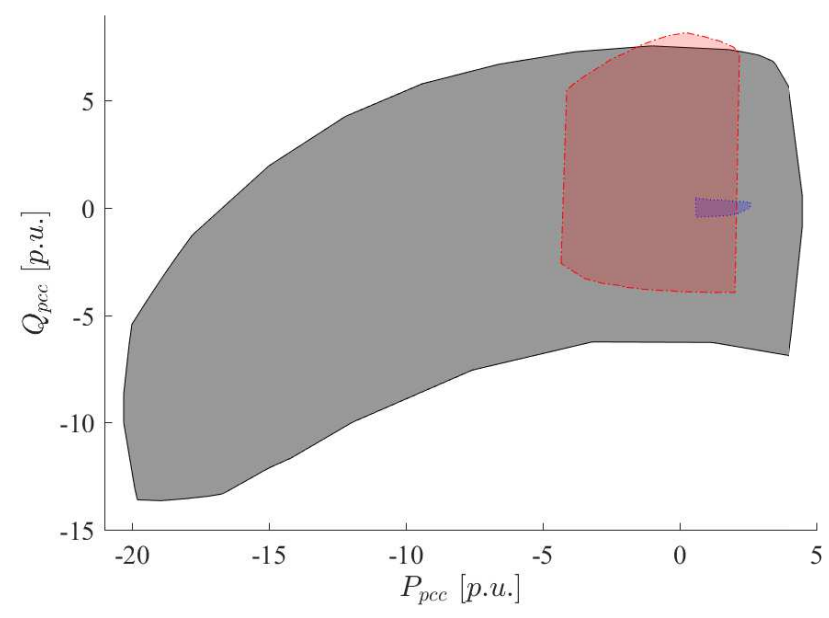

(b) Bus 4062

Fig. 4. TSO desirability surfaces for the buses in the southern area with DSO capability surfaces of considered ADNs

2) Consumption levels are linearly scaled to correspond to variations in Nordpool data. While doing this, it is assumed that the given original values for consumption levels correspond to $80 \%$ consumption levels in Nordpool data.

It is assumed that all the generators vary their active power in the same manner, simultaneously. The same assumption is made for the loads where they simultaneously change the active power keeping the constant original power factors. These assumptions may not correspond to real cases. While simultaneous variations of renewable generation in an ADN may be justified assuming that the whole ADN geograpically belongs to the same meteorological area, the loads at different substations of the ADN may certainly follow different temporal patterns. The simplified approach is used in this paper to illustrate the proccess of obtaining DSO capability surfaces, while for more precise results the spatial variations should be also taken into account.

Using the procedures described in Section II, the TSO desirability surface for the selected buses of the Nordic 32 and DSO capability surfaces for considered ADNs can be obtained. TSO desirability surfaces and DSO capability surfaces for the analyzed system are given in Fig. 2 for the central area buses, Fig. 3 for the northern area buses and Fig. 4 for the southern area buses of the Nordic 32 system. The DSO capability surface of the first analyzed ADN is colored in blue with dotted edges while the second ADN is represented with the red surface with dash-dotted edges. TSO desirability surface for $100 \%$ and $80 \%$ loading scenario are shown as gray surfaces with dashed and full edges respectively. The resulting TSO desirability surface is colored in dark gray as the intersection of the surfaces for all the operating points of the transmission system as shown in Fig. 2. In Fig. 3 and Fig. 4, only the resulting TSO desirability surfaces are shown. For each of the buses, coefficients $D_{\text {flex }}$ and $T_{\text {flex }}$ are calculated using (4) and (5). The results are provided in Table I.

\section{ANALYSIS OF THE RESULTS}

Depending on where the bus is located in the transmission grid, the TSO desirability surface will have a different shape. It can be seen from Fig. 2 that the buses in the central area of the Nordic 32 system have smaller TSO desirability surface compared to the rest of the grid. Consequently, the flexibility of the connected ADNs in this area will have much more value for the TSO. This is also seen from the corresponding $T_{\text {flex }}$ coefficients from Table I for the buses in the central area as they in average have higher values than for the buses in northern and southern area.

The size of a TSO desirability surface also gives information on how strong the respective bus is. Comparing the TSO surfaces for buses 4043 and 4051 or 4022 and 2032, it can be seen that buses 4043 and 4022 are stronger buses than 4051 and 2032. This is an expected result having in mind that buses 4051 and 2032 are located in more remote areas of the grid, while buses 4043 and 4022 are more centrally located in their respective areas. Consequently, buses 4043 and 4022 will have lower $T_{\text {flex }}$ values than buses 4051 and 2032. This means that the flexibility provided by the ADN connected at buses 4051 and 2032 is of a bigger value for the TSO than the flexibility at buses 4043 and 4022 .

The buses 4061 and 4062 located in the southern area have almost the same $T_{\text {flex }}$ coefficients meaning that, from the TSO perspective, provision of the flexibilities from the analyzed ADNs has the same value on both buses. On the other hand, comparing Fig. 4(b) with Fig. 4(a), it can be seen that flexibilities of the second ADN are better used for bus 4062 than 4061 . This means that from the DSO point of view, it is much better to connect to bus 4062 . The same can be seen from the values of $D_{\text {flex }}$ coefficients in Table I as the $D_{\text {flex }}$ value is higher for bus 4062 than 4061.

Comparing the values of $T_{\text {flex }}$ and $D_{\text {flex }}$ coefficients for the first and the second analyzed ADN, it can be concluded that flexibility of ADN 2 has more value for TSO than flexibility of ADN 1. On the other hand, ADN 1 is able 
to provide all of its available flexibilities in any bus of the transmission system. The first reason for this is because the ADN 2 has higher installed power than the ADN 1. The second reason is that the ADN 2 is behaving more like a generator of active power. By analyzing the TSO desirability surfaces of the buses, it can be concluded that the Nordic 32 system can much easier accommodate additional generation of active power than consumption.

The proposed coefficients closely represent how constrained is operation of the ADN and transmission system with regards to their common DSO/TSO interface. If $D_{\text {flex }}$ value at the PCC is low, that would mean that the ADN operates under tightly constrained conditions to sastisfy TSO requirements at the PCC. This induces higher operational costs for DSO. On the other hand, if $D_{\text {flex }}$ value is high, the ADN may operate in loose constrained conditions consequently reducing the DSO operational costs. From TSO point of view, $T_{\text {flex }}$ value gives information on how much DSO operations affect the transmission system operation. High $T_{\text {flex }}$ values indicate that DSO operations may highly affect transmission system operation and low $T_{\text {flex }}$ values mean that the effects are low.

It can be concluded that proposed coefficents reflect the DSO and TSO costs of maintaining connection at the common interface. The cost of the connection to transmission grid per unit of installed power that TSO would charge DSO should be proportional to the $D_{\text {flex }}$ value. On the other hand, the cost of DSO flexibilities provision per unit of installed power to TSO should be proportional to $T_{\text {flex }}$ value.

\section{Discussion}

This paper proposes a method to measure the value of the flexibility of a DSO/TSO interface by introducing flexibility coefficients $D_{\text {flex }}$ and $T_{\text {flex }}$. These coefficients can be calculated from the given DSO capability and TSO desirability surface. These surfaces can be obtained independently by DSO and TSO respectively. This is possible thanks to the assumption that the ADN and the transmission system are connected through the regulating transformer being able to independently control secondary voltage from primary. In this way, the voltage of the PCC point for the ADN is not depending on the state of the transmission system. Therefore, the DSO can calculate its capability without the knowledge of the operating state of the transmission system. For the future work, the effect of this assumption should be investigated and possible improvement of the method should be proposed in case that the assumption is not satisfied. Apart from the correlation between operating states of the systems via voltage of the PCC, other correlations should be investigated as well.

A TSO desirability surface highly depends on the operating point of the transmission system as can be seen from Fig. 2 . To satisfy multiple operating points, the final TSO desirability surface is found as the intersection of the surfaces for each operating point. This is a rather conservative approach having in mind that certain operating points might have higher probability to occur than some others. To account for the probabilities of different operating points, the different areas of the TSO surface should be given different probability values therefore representing more closely actual capability of transmission system to host certain active and reactive power injection. Having the probabilistic information about the surfaces and using probabilistic methods, flexibility coefficients may be calculated more precisely. This extension of the proposed method is subject to a future work.

\section{CONCLUSIONS}

The ongoing changes from conventional to more renewable, distributed generation of power is going to introduce a great deal of challenges for future power systems operation. Some of these challenges can be overcome through the use of power exchange flexibilities of the ADNs connected to the transmission grid. The value of these flexibilities can be assessed in different ways depending on the matter of perspective. Even though a DSO may be very flexible in its adjustment of the exchanged power with the transmission grid, the TSO may find that this flexibility has very small effect on the operation of the transmission system. This paper proposes a method to quantify this flexibility from both TSO and DSO perspective.

To measure the flexibility of DSO/TSO interface appropriately, this paper introduces two flexibility coefficients: $D_{\text {flex }}$ and $T_{\text {flex }}$. The flexibility from the DSO perspective is reflected in the value of $D_{\text {flex }}$ coefficient while $T_{\text {flex }}$ gives the value of the same flexibility from the TSO point of view. Both coefficients are calculated using introduced DSO capability surface and TSO desirability surface defining DSOs capability to provide flexibility and TSO desirability to accept that flexibility. Besides ranking of flexibility of DSO/TSO interface, $D_{\text {flex }}$ coefficient can be used to evaluate TSO connection price for ADN and $T_{\text {flex }}$ coefficient can be used to evaluate DSO price for flexibility provisions to transmission system.

The proposed method is applied on the study case of the Nordic 32 system. Two different ADNs are considered for connection and provision of flexibilities at selected buses of the Nordic 32. It is shown that the introduced flexibility coefficients reflect in a good way the value of the available flexibilities from both TSO and DSO side. Further on, comparing the values of the coefficients as well as different forms of TSO desirability and DSO capability surfaces for different buses, various conclusions about the buses strength and their general behavior can be drawn. In the case of the Nordic 32, one of these conclusions is that the buses in the central area may profit more from the provision of flexibilities than the buses located in the other areas.

\section{REFERENCES}

[1] I. Leisse, "Nysäter-cluster - a new way to regulate reactive power (nysäter-klustret - ett nytt sätt att reglera reaktiv effekt)," May 2017, presentation given at Energiforsk Elkraft 2017 conference.

[2] VDE, Technical rules for operation and planning of electrical networks, Part 1: Interface between transmission and distribution systems, Germany, 2019.

[3] ENTSO-E, Towards smarter grids: Developing TSO and DSO Roles and Interactions for the Benefit of Consumers, 2015. 
[4] - Distributed Flexibility and the value of TSO/DSO cooperation, 2017.

[5] — TSO-DSO Report - An Integrated Apporach to Active System Management, 2019.

[6] T. E. Commision, Commission Regulation (EU) 2016/1388 establishing a Network Code on Demand Connection, The European Commision, 17 August 2016.

[7] E. Hillberg, A. Zegers, B. Herndler, S. Wong, J. Pompee, J.-Y. Bourmaud, S. Lehnhoff, G. Migliavacca, K. Uhlen, I. Oleinikova, H. Pihl, M. Norström, M. Persson, J. Rossi, and G. Beccuti, "Flexibility needs in the future power system," Int. Smart Grid Action Network (ISGAN) Annex 6 Power T\&D Syst., Tech. Rep., March 2019.

[8] G. Migliavacca, M. Rossi, D. Six, M. Džamarija, S. Horsmanheimo, C. Madina, I. Kockar, and J. M. Morales, "Smartnet: H2020 project analysing tso-dso interaction to enable ancillary sevices provision from distribution networks," in Int. Conf. \& Exhibition on Electricity Distribution (CIRED), 2017.

[9] D. A. Contreras and K. Rudion, "Improved assessment of the flexibility range of distribution grids using linear optimization," in Power Syst. Computational Conf., Dublin, Ireland, June 2018.

[10] D. M. Gonzalez, J. Hachenberger, J. Hinker, F. Rewald, U. Häger, C. Rehtanz, and J. Myrzik, "Determination of the time-dependent flexibility of active distribution networks to control their tso-dso interconnection power flow," in Power Syst.Computational Conf., 2018.

[11] J. Silva, J. Sumaili, R. J. Bessa, L. Seca, M. A. Matos, V. Miranda, M. Caujolle, B. Goncer, and M. Sebastian-Viana, "Estimating the active and reactive power flexibility area at the tso-dso interface," IEEE Trans. Power Syst., 2018.

[12] P. Cuffe, P. Smith, and A. Keane, "Capability chart for distributed reactive power resources," IEEE Trans. Power Syst., 2014.

[13] — , "Transmission system impact of wind energy harvesting networks," IEEE Trans. on Sustainable Energy, 2012.

[14] M. Kraiczy, H. Wang, S. Schmidt, F. Wirtz, and M. Braun, "Reactive power management at the transmission-distribution interface with the support of distributed generators -a grid planning approach," IET Generation, Transmission and Distribution, November 2018.

[15] Z. Hagemann and U. Häger, "Reactive power control in distribution networks to minimize the reactive power balance at the point of common coupling," in IEEE PES Innovative Smart Grid Tech. Europe (ISGTEurope), unpublished, Bucharest, Romania, 2019.
[16] S. Stanković and L. Söder, "Identification of reactive power provision boundaries of a distribution grid with dfigs to a transmission grid," in IEEE PES Innovative Smart Grid Tech. Europe (ISGT-Europe), Torino, Italy, 2017.

[17] _ - "Analytical estimation of reactive power capability of a radial distribution system," IEEE Trans. Power Syst., vol. 33, no. 6, pp. 6131 - 6141, November 2018.

[18] The Grid Code, National Grid, 30 September 2016. [Online]. Available: http://www2.nationalgrid.com/uk/industry-information/ electricity-codes/grid-code/the-grid-code/

[19] EirGrid Grid Code, EirGrid, Ireland, June 2019.

[20] H. Gerard, E. I. R. Puente, and D. Six, "Coordination between transmission and distribution system operators in the electricity sector: A conceptual framework," Elsavier Utilities Policy, pp. 40-48, 2018.

[21] G. Valverde and T. V. Cutsem, "Control of dispersed generation to regulate distribution and support transmission voltages," in IEEE PowerTech Conf., 2013.

[22] P. Aristidou, G. Valverde, and T. V. Cutsem, "Contribution of distribution network control to voltage stability: A case study," IEEE Trans. on Smart Grid, 2017.

[23] J. Dragosavac, Žarko Janda, J. V. Milanović, D. Arnautović, and B. Radojičić, "On-line estimation of available generator reactive power for network voltage support," in 8th Mediterranean Conf. on Power Generation, Transmission, Distribution and Energy Conversion (MEDPOWER), 2012

[24] T. Lund, P. Sørensen, and J. Eek, "Reactive power capability of a wind turbine with doubly fed induction generator," Wind Energy, vol. 10, pp. 379-394, April 2007.

[25] G. Valverde and J. J. Orozco, "Reactive power limits in distributed generators from generic capability curves," in IEEE PES General Meeting, July 27-31 2014.

[26] A. Wächter and L. T. Biegler, "On the implementation of a primaldual interior point filter line search algorithm for large-scale nonlinear programming," Mathematical Programming, pp. 25-57, 2006.

[27] T. V. Cutsem and C. Vournas, Voltage Stability of Electric Power Systems, M. A. Pai and A. M. Stankovic, Eds. Springer, 1998.

[28] IEEE Power System Dynamic Performance Committee, "Test systems for voltage stability analysis and security assessment," IEEE Power \& Energy Society, Tech. Rep., August 2015.

[29] Nordpool market data. [Online]. Available: www.nordpoolgroup.com 